\title{
Molecular Characterization of a Novel Integrative Conjugative Element ICEHpa1 in Haemophilus parasuis
} Hua-Run Sunt, Xiao-Die Cuit, Xiao-Kang Liu', Shuai-Hua Li, Kai-Fang Yi, Yu-Shan Pan,
Hua Wu, Li Yuan, Gong-Zheng Hu* and Dan-Dan He*

Department of Pharmacology and Toxicology, College of Animal Science and Veterinary Medicine, Henan Agricultural University, Zhengzhou, China

ICEHpa1 was identified in the genome of a serovar 8 Haemophilus parasuis ST288 isolate YHP170504 from a case of swine lower respiratory tract infection. The aim of the present study was to characterize the integrative conjugative element ICEHpa1 and its multiresistance region. Susceptibility testing was determined by broth microdilution and the complete ICEHpa1 was identified by WGS analysis. The full sequence of ICEHpa1 was analyzed with bioinformatic tools. The presence of ICEHpa1, its circular intermediate and integration site were confirmed by PCR and sequence analysis. Transfer of ICEHpa1 was confirmed by conjugation. ICEHpa1 has a size of 68,922 bp with 37.42\% GC content and harbors 81 genes responsible for replication and stabilization, transfer, integration, and accessory functions, as well as seven different resistance genes [bla Rob-3, $_{2} \operatorname{tet}(B), a p h A 1, \operatorname{str} A, \operatorname{str} B, \operatorname{aac}(6)^{\prime}-l e-a p h\left(2^{\prime}\right)-l a$, and sul2]. Conjugation experiments showed that ICEHpa1 could be transferred to $H$. parasuis V43 with frequencies of $6.1 \times 10^{-6}$. This is the first time a multidrug-resistance ICE has been reported in $H$. parasuis. Seven different resistance genes were located on a novel integrative conjugative element ICEHpa1, which suggests that the ICEHpa1 is capable of acquiring foreign genes and serving as a carrier for various resistance genes.

Keywords: multiresistance, $\boldsymbol{H}$. parasuis, ICE, conjugation, transposon

\section{INTRODUCTION}

The gram-negative bacterium Haemophilus parasuis is the causative agent of Glasser's disease characterized by polyarthritis, fibrinous polyserositis, and meningitis in swine (Oliveira and Pijoan, 2004). The $H$. parasuis infection may cause great economic losses to the global pig industry (Oliveira et al., 2001).

More and more attention has been drawn to the antimicrobial resistance in bacteria from foodproducing animals. In $H$. parasuis, the resistant genes are usually located on small plasmids, in which mob genes ( $m o b A, m o b B$, mobC, mobA-like, mobC-like, and mobA-L) and ISApll are usually identified flanking the resistant genes (Lancashire et al., 2005; Chen et al., 2010; Yang et al., 2013; Li et al., 2015; Moleres et al., 2015). However, no other mobile genetic elements [transposons, integrons, and integrative and conjugative elements (ICEs)] have been found to be associated with the resistant genes in $H$. parasuis. ICEs are self-transmissible mobile elements that are widespread 
among different bacteria (Burrus et al., 2002; Burrus and Waldor, 2004; Lei et al., 2016). ICEs are composed of a set of core genes that are responsible for replication, maintenance, conjugation, recombination, and regulation, with other accessory modules, such as antimicrobial resistance genes (Robinson et al., 2013; Johnson and Grossman, 2015). In addition, ICEs usually have a single insertion site, which is often in the $5^{\prime}$ or $3^{\prime}$ end of a tRNA or other highly conserved genes such as the gene prfC, in the chromosome of their host (Mulvey et al., 2001; Johnson and Grossman, 2015). ICEs, as vehicles for active DNA exchange among different bacteria, contain some specific genes or sites needed for processing their DNA for transfer. Most of these genes are not expressed when the ICE is integrated in the chromosome; however, expression of the genes needed for excision, integration, and conjugation is induced under certain conditions, and the ICE may excise from the host chromosome to form a dsDNA circular intermediate. Some ICE-encoded proteins assemble into a mating pore that is responsible for transferring the ICE. The new host may recognize the origin of transfer (oriT), process the ICE dsDNA to generate a linear ssDNA-protein (T-DNA) through the ICE-encoded relaxase, and pump the T-DNA into the recipient. Then the ICE was recombined into the new host chromosome through an ICE-encoded integrase (Toleman and Walsh, 2011; Johnson and Grossman, 2015; Wright et al., 2015).

Quite a few ICEs have been identified in Pasteurellaceae, such as Haemophilus influenzae, Pasteurella multocida, Mannheimia haemolytica, and Actinobacillus pleuropneumoniae (Juhas et al., 2007; Brenner et al., 2012; Eidam et al., 2015; Bossé et al., 2016; Li Y. et al., 2018). However, no complete multidrug-resistance ICE in $H$. parasuis has been described in detail to date. In this study, we identified ICEHpa1, a novel ICE carrying multiple resistance genes, in the chromosome of a serovar $8 \mathrm{H}$. parasuis ST288 isolate YHP170504, in a feedlot from Henan, China, in 2017.

\section{MATERIALS AND METHODS}

\section{Bacterial Strains and Susceptibility Testing}

The strain YHP170504 was obtained from a case of swine lower respiratory tract infection in a feedlot from Henan, China, in 2017. Owing to the unavailability of an approved method for $H$. parasuis, MICs of $H$. parasuis isolates were determined using broth microdilution method following CLSI standard (Clinical and Laboratory Standards Institute [CLSI], 2018) for A. pleuropneumoniae. The antimicrobial agents tested were oxytetracycline, doxycycline, ampicillin, amoxicillin, ceftiofur, cefquinome, enrofloxacin, streptomycin, gentamicin, tilmicosin, tylosin, florfenicol, sulfamethoxazole/trimethoprim (19/1), lincomycin, and colistin. A. pleuropneumoniae ATCC27090 and Escherichia coli ATCC 25922 were used as control strains.

\section{WGS and Analysis}

Total genomic DNA of strain YHP170504 was extracted using the TIANamp Bacteria DNA Kit (TIANGEN, Beijing, China) and subjected to WGS using Illumina Nextseq 500 and the Oxford Nanopore Technologies (ONT) MinION platforms. Sequencing reads including short-read and long-read data were assembled using Unicycler 0.4.4 with the hybrid strategy (Wick et al., 2017; Li R. et al., 2018). The complete sequence of ICEHpa1 was initially annotated using the RAST server ${ }^{1}$ and corrected manually. Comparison analysis was conducted using the genome comparison visualizer Easyfig.

\section{Confirmation of the Circular Extrachromosomal Form of the ICE and Conjugal Transfer of ICEHpa1}

Chromosomal insertion sites were confirmed by PCR in YHP170504 and transconjugants. For the $5^{\prime}$ junction, primers F1 and R1 were designed to amplify a 515-bp fragment from upstream of the ICEHpal insertion to a sequence within the $5^{\prime}$ end of ICEHpa1. For the $3^{\prime}$ insertion, primers F2 and R2 were designed to amplify a $458 \mathrm{bp}$ fragment from within the $3^{\prime}$ end of ICEHpal insertion to a sequence of the downstream of the ICEHpal insertion. To confirm the extrachromosomal circular form of the ICE, outward-facing primers ICE-out-F and ICE-out-R were used.

To investigate self-transfer ability of the ICEHpa1, the conjugation assay was performed using the $H$. parasuis strain YHP170504 as the donor and $H$. parasuis V43 (rifampicin resistance) as the recipient. The serovar $4 \mathrm{H}$. parasuis ST170 isolate V43 was from the strain collection of our laboratory, and the rifampicin-resistant mutant of this strain was generated by selection on Tryptic Soy Agar (TSA) plates supplemented with $10 \%$ fetal bovine serum, $10 \mathrm{mg} / \mathrm{L}$ nicotinamide adenine dinucleotide (NAD), and increasing rifampicin concentration. For the conjugation assay, overnight cultures of donor and recipient strains grown in Tryptic Soy Broth supplemented with $10 \%$ fetal bovine serum and $10 \mathrm{mg} / \mathrm{L}$ NAD were mixed (1:5) and incubated for $4 \mathrm{~h}$ at $37^{\circ} \mathrm{C}$. Bacterial cultures were spread on TSA plates supplemented with $10 \%$ fetal bovine serum, $10 \mathrm{mg} / \mathrm{L} \mathrm{NAD}$, oxytetracycline $(8 \mathrm{mg} / \mathrm{L})$, and rifampicin $(100 \mathrm{mg} / \mathrm{L})$, incubated at $37^{\circ} \mathrm{C}$ for $24 \mathrm{~h}$. Then the transconjugants were screened on the plates. The conjugation frequency was calculated as the number of transconjugants per donor. All the transconjugants were confirmed with PCR using the primers virB4-F and virB4$\mathrm{R}$, susceptibility testing, and MLST. All the primers used are listed in Table 1.

\section{Serotyping and Multilocus Sequence Typing (MLST)}

The strain YHP170504, V43, and the transconjugant V43::ICEHpal1 were typed by serotyping and MLST. Serovars of the strains were determined using the primers Howell previously described (Howell et al., 2015). Seven housekeeping genes (atpD, infB, $m d h, r p o B, 6 p g d, g 3 p d$, and $f r d B$ ) were amplified and sequenced as described previously (Mullins et al., 2013), after registration of sequences at https://pubmlst.org/hparasuis/ for assignment of allele numbers and STs; data were analyzed using software available on the website.

\footnotetext{
${ }^{1}$ http://rast.nmpdr.org
} 
TABLE 1 | PCR primers used in this study.

\begin{tabular}{|c|c|c|c|c|}
\hline Target & Primer & Sequence $5^{\prime}-3^{\prime}$ & Amplicon size (bp) & Annealing temperature $\left({ }^{\circ} \mathrm{C}\right)$ \\
\hline \multirow[t]{2}{*}{$5^{\prime}$ junction of ICEHpa1 } & $\mathrm{F} 1$ & ATGTGGTGAATATITAACTA & 515 & 53 \\
\hline & $\mathrm{R} 1$ & GGTGCAGAATCTTCAATATG & & \\
\hline \multirow[t]{2}{*}{$3^{\prime}$ junction of ICEHpa1 } & $\mathrm{F} 2$ & TCTAGACTITACAAGAAAAC & 458 & 50 \\
\hline & $\mathrm{R} 2$ & AGCTACATTGACTATAACGC & & \\
\hline Circular form and insertion & ICE-out-F & CGAGTGAAAAATTCATACAA & 712 & 47 \\
\hline sites of the ICEHpa1 & ICE-out-R & TGGTTCCCTATITCTAGCC & & \\
\hline \multirow[t]{2}{*}{ virB4 } & virB4-F & CAATACAAGCCATCGCTATC & 393 & 55 \\
\hline & virB4-R & TाTGTCTTCGAATAGACCAC & & \\
\hline \multirow[t]{2}{*}{ Circular form of the Tn6742 } & Tn-out-F & GGCTATTTCACCCACGCACT & 1036 & 55 \\
\hline & Tn-out-R & TGAGTACTTCCTACCGACAT & & \\
\hline
\end{tabular}

\section{Evolutionary Analyses of the Integrase}

The integrase, an important core gene of ICE, is needed for both integration and excision. Evolutionary analyses were conducted in MEGA7 (Kumar et al., 2016) and the analysis involved 11 integrases complete sequences of eight ICEs from Pasteurellaceae. Initial trees for the heuristic search were obtained automatically by applying Neighbor-Join and BioNJ algorithms to a matrix of pairwise distances estimated using the maximum composite likelihood approach and then selecting the topology with superior log-likelihood value (Kumar et al., 2016).

\section{Nucleotide Sequence Accession Number}

The complete sequences of the chromosome and the ICEHpal in $H$. parasuis YHP170504 have been submitted to GenBank with the following accession numbers: CP054198 and MN844034.

\section{RESULTS AND DISCUSSION}

The strain YHP170504 was serotyped as serovar 8, belonging to ST288. Also, it exhibited high MICs of oxytetracycline (64 mg/L), doxycycline $(8 \mathrm{mg} / \mathrm{L})$, ampicillin $(64 \mathrm{mg} / \mathrm{L})$, amoxicillin $(64 \mathrm{mg} / \mathrm{L})$, gentamicin $(256 \mathrm{mg} / \mathrm{L})$, streptomycin (128 mg/L), sulfamethoxazole/trimethoprim $(513 / 27 \mathrm{mg} / \mathrm{L})$, and enrofloxacin $(8 \mathrm{mg} / \mathrm{L})$.

The chromosome of $H$. parasuis YHP170504 is 2,520,015 bp long with a GC content of $39.64 \%$. Sequence analysis showed that the genome harbors seven resistance genes including the $\beta$-lactamase-encoding gene $b l a_{\mathrm{Rob}-3}$, tetracycline resistance gene tet(B), the aminoglycoside resistance genes (aphA1, strA, and $\operatorname{str} B$ ), aminoglycoside and fluoroquinolone resistance gene $\left[a a c(6)^{\prime}-I e-a p h\left(2^{\prime}\right)-I a\right]$, and sulfonamide resistance gene sul2. WGS analysis showed that all seven resistance genes were located on a novel integrative conjugative element, designated as ICEHpa1 (Figure 1A) according to the nomenclature of ICEs ${ }^{2}$.

ICEHpal $(68,922 \mathrm{bp}$, corresponding to bases $1,511,383-$ $1,580,304$ in GenBank accession number CP054198 or bases 5938-74,859 in GenBank accession number MN844034), a novel ICE with a lower GC content (37.42\%), differing from the GC content $(39.64 \%)$ of entire genome of H. parasuis YHP170504,

${ }^{2}$ https://db-mml.sjtu.edu.cn/ICEberg/ was integrated into the tRNA ${ }^{\mathrm{Leu}}$, a common insertion site for ICEs in other species of the family Pasteurellaceae (Juhas et al., 2007; Brenner et al., 2012; Eidam et al., 2015; Bossé et al., 2016). WGS analysis showed that the ICEHpal was flanked by two tRNA ${ }^{\text {Leu }}$ copies (a truncated copy and an intact copy, Figure 1A). The presence of an extrachromosomal circular form of ICEHpal was confirmed in YHP170504 using primers ICE-out-F and ICE-out-R. Sequencing of the closed circular form of ICEHpal showed that the junction (attP sequence) sequence was formed by $56 \mathrm{bp}$ imperfect direct repeats (Figure 1B).

PCR assays and susceptibility testing confirmed the presence and activity of the ICEHpal-associated resistance genes in the $H$. parasuis V43. The transconjugant H. parasuis V43::ICEHpal1 showed, in comparison with $\mathrm{H}$. parasuis V43, increased MICs of oxytetracycline (from $<0.5$ to $32 \mathrm{mg} / \mathrm{L}$ ), doxycycline (from $<0.5$ to $8 \mathrm{mg} / \mathrm{L}$ ), ampicillin (from 1 to $64 \mathrm{mg} / \mathrm{L}$ ), amoxicillin (from 1 to $64 \mathrm{mg} / \mathrm{L}$ ), gentamicin (from $<0.5$ to $128 \mathrm{mg} / \mathrm{L}$ ), streptomycin (from 2 to $128 \mathrm{mg} / \mathrm{L}$ ), sulfamethoxazole/trimethoprim (from $1.9 / 0.1$ to $513 / 27 \mathrm{mg} / \mathrm{L}$ ), and enrofloxacin (from $<0.5$ to $4 \mathrm{mg} / \mathrm{L}$ ). Also, the transconjugant $H$. parasuis V43::ICEHpall showed the same serovar and ST as $H$. parasuis V43. Although only traD, traG, virB4, pilT, and pill genes encoding components of the type IV secretion system were identified, many other conjugal transfer proteins and membrane proteins were present in ICEHpa1, which may also be involved in the conjugal transfer and responsible for the host specificity of the ICE. The ICEHpal could be transferred to $H$. parasuis V43 at a low frequency of conjugation, with $6.1 \times 10^{-6}$ transconjugants per donor. Our results revealed that ICEHpal has the selftransmissible capacity to facilitate the dissemination of the resistance genes.

Sequence analysis indicated that the insertion point of ICEHpa1 in the transconjugant was located in a tRNA ${ }^{\text {Leu }}$. Also, the tRNA ${ }^{\mathrm{Leu}}$ locus, in which the ICEHpal was inserted in the $H$. parasuis V43, showed the same sequence as the one in the H. parasuis YHP170504 (Figure 1B). The tRNA ${ }^{\mathrm{Leu}}$ into which ICEHpal was inserted in H. parasuis YHP170504 was flanked on one side by a peptide transporter and on the other side by a hypothetical protein. A comparison between the genome of $H$. parasuis YHP170504 and another similar genome of Glaesserella parasuis (H. parasuis) D74 showed that the genome 


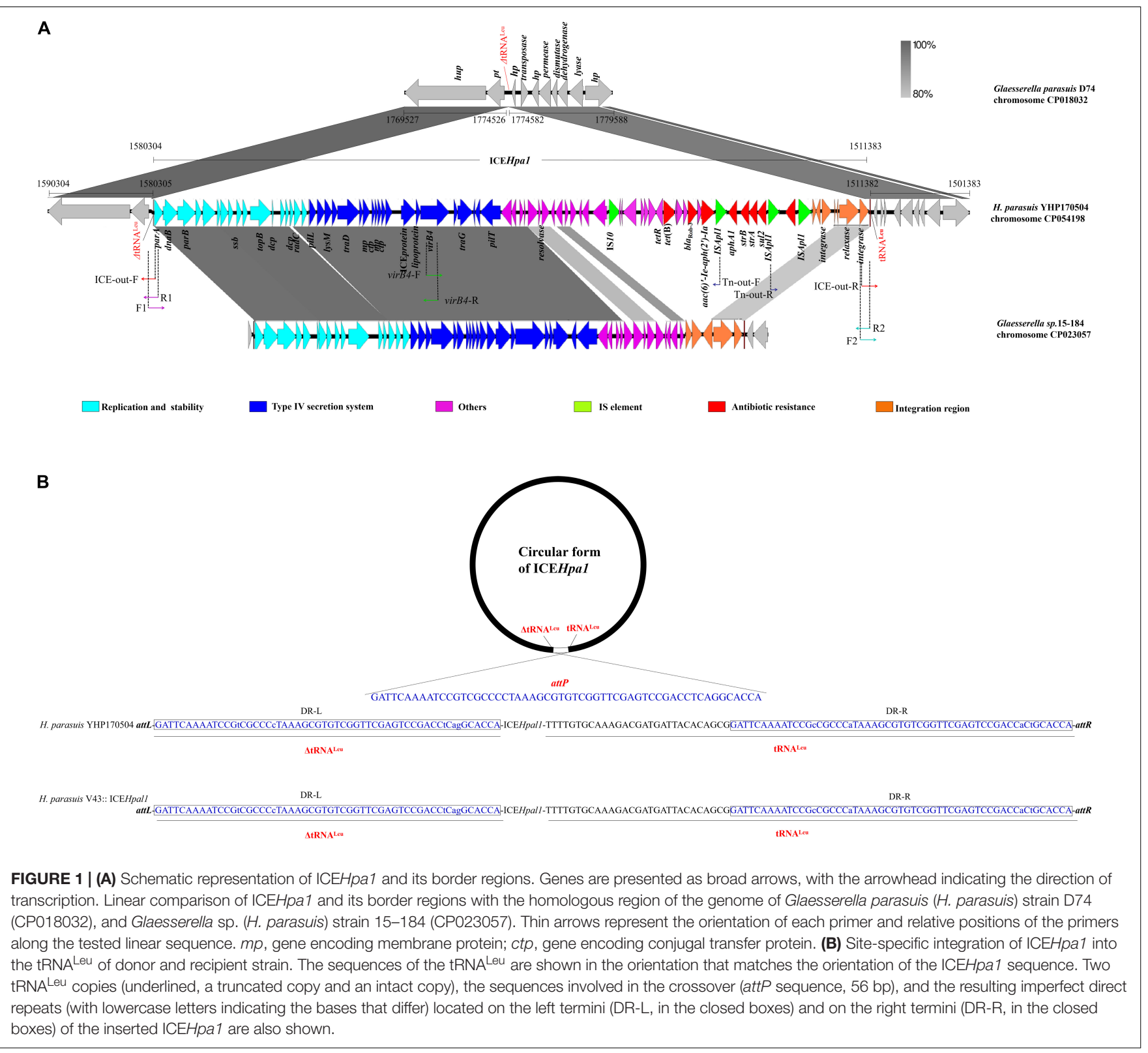

fragment flanking the left border and right border regions of the ICEHpa1 in YHP170504 had high identity to the D74, but a 55-bp truncated tRNA ${ }^{\text {Leu }}$ was replaced by the ICEHpal insertion in YHP170504. Similarly, a peptide transporter and a hypothetical protein were found immediately up- and downstream of the tRNA $^{\text {Leu }}$ in Glaesserella parasuis D74. The result revealed the strain Glaesserella parasuis D74 may serve as a potential recipient for acquiring ICEHpa1.

A total of 81 genes flanked by two tRNA ${ }^{\text {Leu }}$ copies were identified within ICEHpal, of which 17 coded for the putative replication, 20 for type IV secretion system (T4SS), 7 for integration, 4 for transposases of IS elements (an IS10 and three copies of ISApl1), and 7 for resistance genes including the $b l a_{\text {Rob-3 }}, \operatorname{tet}(\mathrm{B}), \operatorname{aph} A 1, \operatorname{str} A, \operatorname{str} B, \operatorname{aac}(6)^{\prime}-I e-a p h\left(2^{\prime}\right)-I a$, and sul2. Comparative sequence analysis (Figure 1A) showed that the complete sequence of ICEHpal shared only $63 \%$ identity (the highest rate of match) with the region of Glaesserella sp. (H. parasuis) 15-184 chromosome, differing clearly from the previous reports about the ICEs from the other Pasteurellaceae species (Juhas et al., 2007; Brenner et al., 2012; Eidam et al., 2015; Bossé et al., 2016; Li Y. et al., 2018).

ICEHpa1 contains five components (replication and stabilization, T4SS, antimicrobial resistance region, integration and accessory region) (Figure 1A), whose replication and T4SS region shared $98.18 \%$ identity with the corresponding region of Glaesserella sp. 15-184 chromosome. The accessory region and integration region (including two integrase genes and a relaxase gene) exhibited only partial homology to corresponding region of Glaesserella sp. 15-184 chromosome (69\% coverage with $96.41 \%$ identity and $61 \%$ coverage with $85.05 \%$ identity, respectively) 
(Figure 1A). Similar to ICEPmu1 in P. multocida (Brenner et al., 2012), two integrases were found in ICEHpa1. Identity of $47.35 \%$ (93\% coverage) was seen when the amino acid sequences of these two integrases were aligned. Comparative sequence analysis revealed that these two integrases are tyrosine recombinases of the Xer family, which are responsible for the integration by site-specific recombination. The integrase 1 in ICEHpal, belonging to tyrosine recombinase XerD, shared 93.98\% (98\% coverage) amino acid identity to integrase (Pmu_02700) of ICEPmu1 (CP003022). The integrase 2 in ICEHpa1, belonging to tyrosine recombinase XerC, shared $100 \%$ (100\% coverage) amino acid identity to integrase of Glaesserella parasuis strain F9 (KEZ23006.1). However, both of them differed from the integrases reported in ICEs from Pasteurellaceae according to the maximum-likelihood tree obtained by using MEGA 7 software (Figure 2). Other experiments are necessary to show which of them or if both are responsible for the integration of ICEHpal.

The multiresistance region (Figure $\mathbf{3 A}$ ) contains three segments harboring seven different resistance genes. The first segment harboring tet(B) was characterized by a truncated transposon Tn10, which shows 99\% identity with the corresponding region of the Tn10 transposon. The second segment carries two resistance genes, bla Rob-3 and $a a c(6)^{\prime}-I e-$ $a p h\left(2^{\prime}\right)-I a$. Notably, although this region showed $99 \%$ identity with the corresponding region of the $H$. parasuis pQY431 complete sequence, its $b l a_{\text {Rob }}$ gene significantly differed from its counterpart in $H$. parasuis pQY431. Compared with the Bla $a_{\text {Rob }}$ protein encoded by bla $a_{\text {Rob-1 }}$ from the $H$. parasuis pQY431, two alanine residues [leucine $(\mathrm{L})$ and threonine $(\mathrm{T})$ ] were added

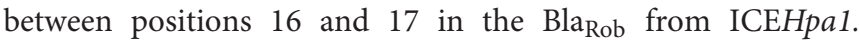

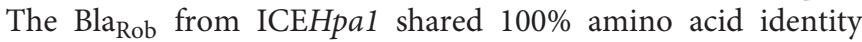
with the Bla $\mathrm{Rob}_{3}$ from Moraxella pluranimalium CCUG 54913 (NG059331). Resistance to $\beta$-lactam antibiotics in $H$. parasuis is conferred by two potential mechanisms: mutations in the ftsI gene encoding PBP3 and/or production of $\beta$-lactamases. WGS analysis showed that no point mutation was found in the ftsI gene and no other bla gene was detected in $H$. parasuis YHP170504 except $b l a_{\text {Rob-3, }}$, which suggests that the bla $a_{\text {Rob-3 }}$ gene conferred resistance to ampicillin and amoxicillin. To our knowledge, this is the first report of $b l a_{\mathrm{Rob}-3}$ gene in Pasteurellaceae species. The third segment harboring a resistance module aphA1-strB-strA-sul2, which is flanked by two ISApl1 elements oriented in the same direction, was regarded as a putative small transposon designated Tn6742 (Figure 3B). To confirm the excision and cyclization of this structure, PCR was conducted using the primers, Tn-out-F and Tn-out-R. The result showed this structure can be looped out, which indicated ISApl1 might accelerate the dissemination of the module aphA1strB-strA-sul2. ISApl1 has been reported to produce a 2 bp direct duplication GG at its integration site (Tegetmeyer et al., 2008). The 2 bp direct duplication GG was detected upstream of the left-hand copy and downstream of the right-hand copy

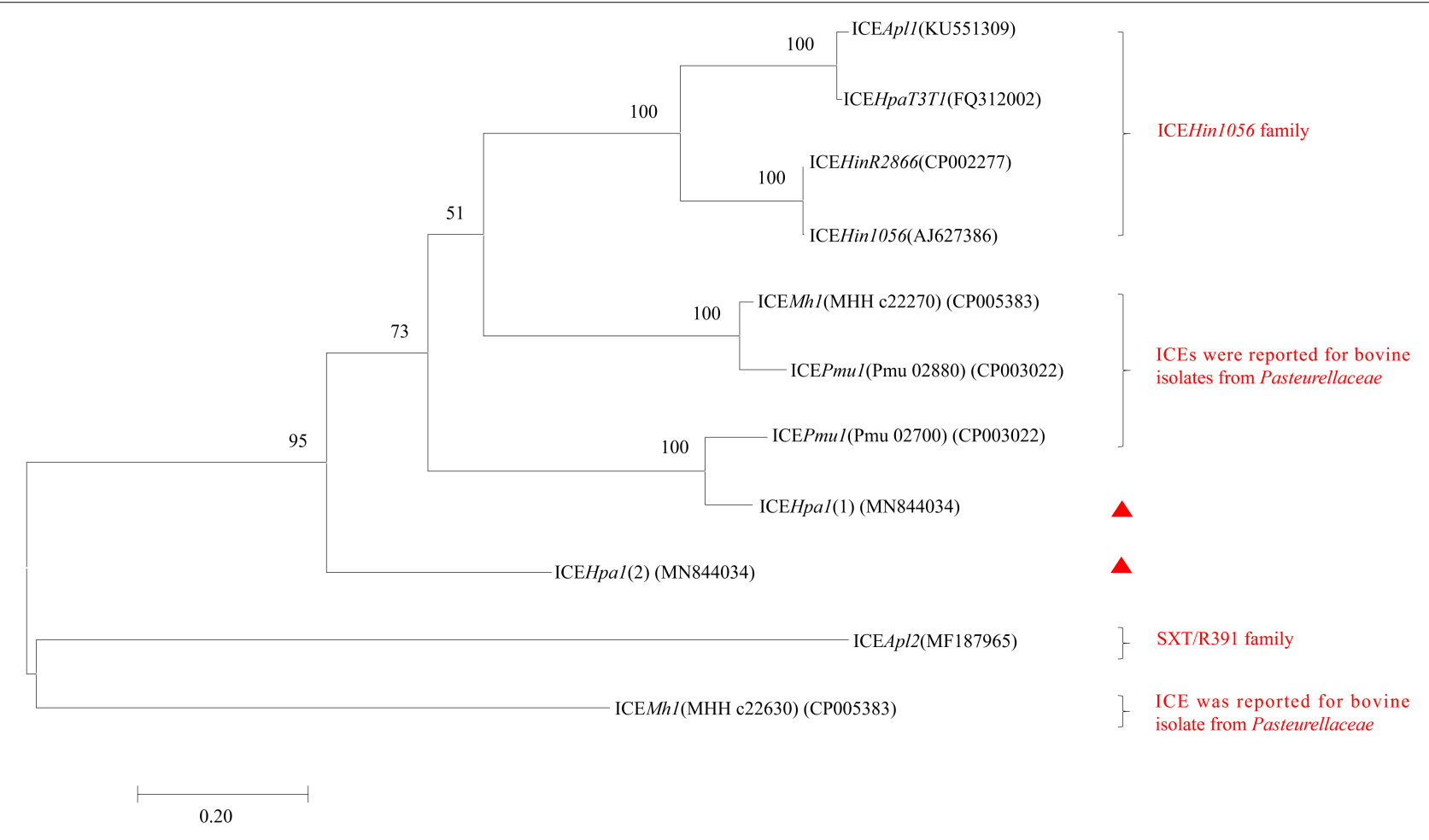

FIGURE 2 | Maximum-likelihood phylogenetic tree based on 11 integrases complete sequences of 8 ICEs from Pasteurellaceae by using MEGA7. Two integrases of ICEHpa1 are indicated by red triangles. The percentage of trees in which the associated taxa clustered together is shown next to the branches. The tree is drawn to scale, with branch lengths measured in the number of substitutions per site. All positions containing gaps and missing data were eliminated. Bootstrap analysis was performed with 1000 replications. Bar, 0.2 substitution per nucleotide position. 
A

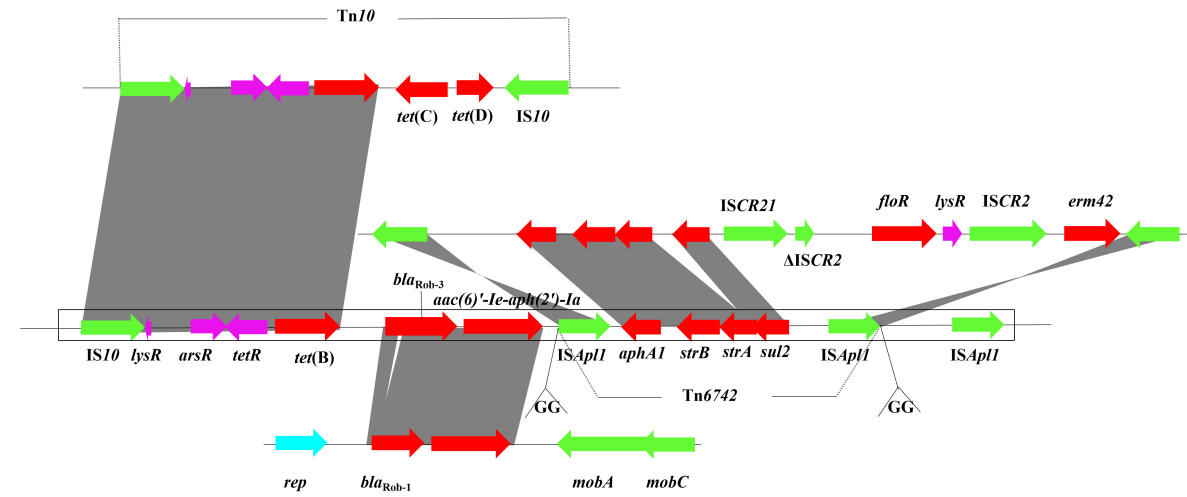

Shigella flexneri transposon Tn10 AF162223
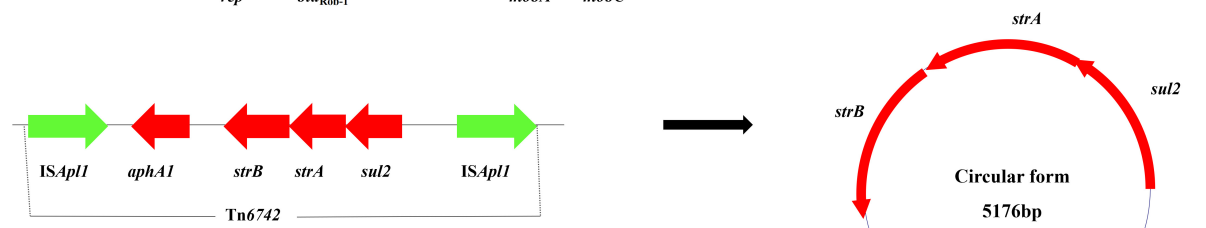
KC405065

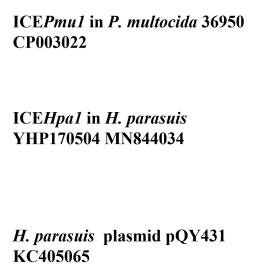

B

$\square$ Replication and stability
Type IV secretion system

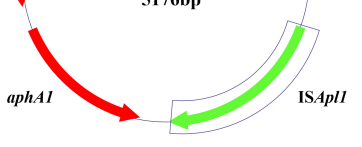

Antibiotic resistance

Integration region

FIGURE 3 | (A) Schematic representation the resistance gene region of ICEHpa1. Genes are presented as arrows, with the arrowhead indicating the direction of transcription. Linear comparison of resistance gene region of ICEHpa1 with the homologous region of the transposon Tn10 of Shigella flexneri (AF162223), plasmid pQY431 of H. parasuis (KC405065), and ICEPmu1 of P. multocida 36950 (CP003022). (B) The circular form of the ISAp/1-aphA1-strB-strA-sul2-ISAp/1 segment in ICEHPa1.

of ISApl1, which suggested that the transposon Tn6742 was reassembled into the host chromosome via ISApl1-mediated insertion rather than homologous recombination. However, the formation of a similar structure ISApl1-aphA1-strB-strAsul2-ISCR21-DISCR2-floR-ISCR2-erm42-ISApl1 in ICEPmu1 from $P$. multocida was proved to insert the chromosome by producing a 2-bp direct duplication GT rather than GG (Brenner et al., 2012). In addition, all the four resistance genes $a p h A 1, \operatorname{str} B, \operatorname{str} A$, and $\operatorname{sul} 2$ oriented in the same direction, which is opposite the direction of the two ISApll. Compared with that, the four genes and two ISApl1, all oriented in the same direction, were identified in ICEPmu1 from P. multocida (Brenner et al., 2012). Additional experiments are necessary to show the role of the third ISApl1 in the transmission of these resistance genes.

\section{CONCLUSION}

In summary, a novel ICE was identified from a serovar $8 \mathrm{H}$. parasuis ST288 isolate. To our knowledge, this is the first time a multidrug-resistance ICE has been reported in $H$. parasuis. A total of seven different resistance genes were located on the ICEHpa1, which suggests that the ICEHpal may act as a reservoir for various resistance genes. Therefore, more research and effective surveillance is needed to monitor the dissemination of multidrug-resistance ICEs.

\section{DATA AVAILABILITY STATEMENT}

The datasets presented in this study can be found in online repositories. The names of the repository/repositories and accession number(s) can be found in the article/supplementary material.

\section{ETHICS STATEMENT}

This study was carried out in accordance with the guidelines of Henan Agricultural University Animal Ethics Committee. The owners of the farm animals from which samples were taken gave permission for their animals to be used in this study.

\section{AUTHOR CONTRIBUTIONS}

G-ZH conceived and designed the experiments. H-RS, X-DC, $\mathrm{X}-\mathrm{KL}$, and K-FY produced the data. H-RS, Y-SP, D-DH, HW, LY, and G-ZH analyzed the data. H-RS and G-ZH wrote the manuscript. All authors contributed to the article and approved the submitted version.

\section{FUNDING}

This study was financed by the National Key Research and Development Program of China (2016YFD05101304). 


\section{REFERENCES}

Bossé, J. T., Li, Y., Roberto, F. C., Chaudhuri, R. R., Rogers, J., Holden, M. T. G., et al. (2016). ICEApl1, an integrative conjugative element related to ICEHin1056, identified in the pig pathogen Actinobacillus pleuropneumoniae. Front. Microbiol. 7:810. doi: 10.3389/fmicb.2016.00810

Brenner, M. G., Kristina, K., Sweeney, M. T., Elzbieta, B., Heiko, L., Rolf, D., et al. (2012). ICEPmu1, an integrative conjugative element (ICE) of Pasteurella multocida: analysis of the regions that comprise 12 antimicrobial resistance genes. Jo. Antimicrob. Chemother. 67, 84-90. doi: 10.1093/jac/dkr406

Burrus, V., Pavlovic, G., Decaris, B., and Guedon, G. (2002). Conjugative transposons: the tip of the iceberg. Mol. Microbiol. 46, 601-610. doi: 10.1046/ j.1365-2958.2002.03191.x

Burrus, V., and Waldor, M. K. (2004). Shaping bacterial genomes with integrative an conjugative elements. Res. Microbiol. 155, 376-386. doi: 10.1016/j.resmic. 01.012

Chen, L. P., Cai, X. W., Wang, X. R., Zhou, X. L., Wu, D. F., Xu, X. J., et al. (2010). Characterization of plasmid-mediated lincosamide resistance in a field isolate of Haemophilus parasuis. J. Antimicrob. Chemother. 65, 2256. doi: 10.1093/jac/ dkq304

Clinical and Laboratory Standards Institute [CLSI] (2018). "Performance standards for antimicrobial disk and dilution susceptibility tests for bacteria isolated from animals," in CLSI supplement VET08, 4th Edn (Wayne, PA: CLSI).

Eidam, C., Poehlein, A., Leimbach, A., Michael, G. B., Kadlec, K., Liesegang, H., et al. (2015). Analysis and comparative genomics of ICEMh1, a novel integrative and conjugative element (ICE) of Mannheimia haemolytica. Jo. Antimicrob. Chemother. 70, 93-97. doi: 10.1093/jac/dku361

Howell, K. J., Peters, S. E., Wang, J., Hernandez-Garcia, J., Weinert, L. A., Luan, S. L., et al. (2015). Development of a multiplex PCR assay for rapid molecular serotyping of Haemophilus parasuis. J. Clin. Microbiol. 53, 3812-3821. doi: 10.1128/jcm.01991- 15

Johnson, C. M., and Grossman, A. D. (2015). Integrative and Conjugative Elements (ICEs): what They Do and How They Work. Ann. Rev. Genet. 49, 577-601. doi: 10.1146/annurev-genet-112414-055018

Juhas, M., Power, P. M., Harding, R. M., Ferguson, D. J. P., Dimopoulou, I. D., Elamin, A. R. E., et al. (2007). Sequence and functional analyses of Haemophilus spp. genomic islands. Genome Biol. 8:R237. doi: 10.1186/gb-2007-8-11r237

Kumar, S., Stecher, G., and Tamura, K. (2016). MEGA7: molecular evolutionary genetics analysis version 7.0 for bigger datasets. Mol. Biol. Evol. 33, 1870-1874. doi: 10.1093/molbev/msw054

Lancashire, J. F., Terry, T. D., Blackall, P. J., and Jennings, M. P. (2005). Plasmid-encoded tet B tetracycline resistance in Haemophilus parasuis. Antimicrob. Agents Chemother. 49:1927. doi: 10.1128/AAC.49.5.1927-1931. 2005

Lei, C. W., Zhang, A. Y., Wang, H. N., Liu, B. H., Yang, L. Q., and Yang, Y. Q. (2016). Characterization of SXT/R391 integrative and conjugative elements in Proteus mirabilis isolates from food-producing animals in China. Antimicrob. Agents Chemother. 60:1935. doi: 10.1128/AAC.02852-15

Li, B., Zhang, Y., Wei, J., Shao, D., Liu, K., Shi, Y., et al. (2015). Characterization of a novel small plasmid carrying the florfenicol resistance gene floR in Haemophilus parasuis. J. Antimicrob. Chemother. 70, 3159-3161. doi: 10.1093/ $\mathrm{jac} / \mathrm{dkv} 230$

Li, R., Xie, M., Dong, N., Lin, D., Yang, X., Wong, M. H. Y., et al. (2018). Efficient generation of complete sequences of MDR-encoding plasmids by rapid assembly of MinION barcoding sequencing data. Gigascience 7, 1-9. doi: 10. 1093/gigascience/gix132
Li, Y., Li, Y., Crespo, R. F., Leanse, L. G., Langford, P. R., and Bosse, J. T. (2018). Characterization of the Actinobacillus pleuropneumoniae SXTrelated integrative and conjugative element ICEApl2 and analysis of the encoded FloR protein: hydrophobic residues in transmembrane domains contribute dynamically to florfenicol and chloramphenicol efflux. J. Antimicrob. Chemother. 73, 57-65. doi: 10.1093/jac/dkx342

Moleres, J., Santos-López, A., Lázaro, I., Labairu, J., Prat, C., Ardanuy, C., et al. (2015). Novel blaROB-1-bearing plasmid conferring resistance to $\beta$-lactams in Haemophilus parasuis isolates from healthy weaning pigs. Appl. Environ. Microbiol. 81, 3255-3267. doi: 10.1128/AEM.03865-3814

Mullins, M. A., Register, K. B., Brunelle, B. W., Aragon, V., Galofré-Mila, N., Baylese, D., et al. (2013). A curated public database for multilocus sequence typing (MLST) and analysis of Haemophilus parasuis based on an optimized typing scheme. Vet. Microbiol. 162, 899-906. doi: 10.1016/j.vetmic.2012.11.019

Mulvey, M., Martin, M., Alfa, M., and Ng, R. M. K. (2001). Multiplex PCR for the detection of tetracycline resistant genes. Mol. Cell Probes. 15, 209-215. doi: 10.1006/mcpr.2001.0363

Oliveira, S., Galina, L., and Pijoan, C. (2001). Development of a PCR test to diagnose Haemophilus parasuis infections. J. Vet. Diagn. Invest. 13, 495-501. doi: $10.1177 / 104063870101300607$

Oliveira, S., and Pijoan, C. (2004). Haemophilus parasuis: new trends on diagnosis epidemiology and control. Vet. Microbiol. 99, 1-12. doi: 10.1016/j.vetmic.2003. 12.001

Robinson, E., Didelot, X., Hood, D., and Crook, D. (2013). Comparative phylogenetics of ICEHin1056 family reveals deep evolutionary associations of mobile genetic elements responsible for transfer of antibiotic resistance genes. Lancet 381(Suppl.1):S93. doi: 10.1016/S0140-6736(13)60533-4

Tegetmeyer, H. E., Jones, S. C. P., Langford, P. R., and Baltes, N. (2008). ISApl1, a novel insertion element of Actinobacillus pleuropneumoniae, prevents ApxIVbased serological detection of serotype 7 strain AP76. Vet. Microbiol. 128, 342-353. doi: 10.1016/j.vetmic.2007.10.025

Toleman, M. A., and Walsh, T. R. (2011). Combinatorial events of insertion sequences and ICE in Gram-negative bacteria. FEMS Microbiol. Rev. 35, 912 935. doi: 10.1111/j.1574-6976.2011.00294.x

Wick, R. R., Judd, L. M., Gorrie, C. L., and Holt, K. E. (2017). Unicycler: resolving bacterial genome assemblies from short and long sequencing reads. PLoS Comput. Biol. 13:e1005595. doi: 10.1371/journal.pcbi.1005595

Wright, L. D., Johnson, C. M., Grossman, A. D., and Viollier, P. H. (2015). Identification of a single strand origin of replication in the integrative and conjugative element ICEBs1 of Bacillus subtilis. PLoS Genet. 11:e1005556. doi: 10.1371/journal.pgen.1005556

Yang, S. S., Sun, J., Liao, X. P., Liu, B. T., Li, L. L., Li, L., et al. (2013). Co-location of the $\operatorname{erm}(\mathrm{T})$ gene and blaROB-1 gene on a small plasmid in Haemophilus parasuis of pig origin. J. Antimicrob. Chemother. 68, 1930-1932. doi: 10.1093/ jac/dkt112

Conflict of Interest: The authors declare that the research was conducted in the absence of any commercial or financial relationships that could be construed as a potential conflict of interest.

Copyright (C) 2020 Sun, Cui, Liu, Li, Yi, Pan, Wu, Yuan, Hu and He. This is an open-access article distributed under the terms of the Creative Commons Attribution License (CC BY). The use, distribution or reproduction in other forums is permitted, provided the original author(s) and the copyright owner(s) are credited and that the original publication in this journal is cited, in accordance with accepted academic practice. No use, distribution or reproduction is permitted which does not comply with these terms. 